Journal of Case Reports 2018;8(4):271-274

\title{
Swelling on the Arm: An Unusual Presentation of Microfilaria
}

\author{
Supriya M Paranjpe, Avani H Koticha, Preeti R Mehta \\ Department of Microbiology, Seth G.S. Medical College \& KEM Hospital, Parel, Mumbai, India.
}

Corresponding Author:

Dr Supriya Paranjpe

Email: supriyamparanjpe@gmail.com

This is an Open Access article distributed under the terms of the Creative Commons Attribution License (creativecommons.org/ licenses/by/3.0).

Received

Accepted

July 8, 2018

November 20, 2018

Published

\begin{abstract}
Background: Bancroftian filariasis is a tropical and sub-tropical disease caused by Wuchereria bancrofti and transmitted by the culex mosquitoes. The diagnosis of it is conventionally made by demonstrating microfilariae in the peripheral blood smear. Case Report: We hereby report an unusual case of bancroftian filariasis in an adult male presenting with a painless sub-cutaneous nodule on the antero-medial aspect of the right arm. Conclusion: Our aim is to highlight the chances of finding microfilariae in an unsuspected case at an unusual site.
\end{abstract}

Keywords: Culex, Filarisis, Male, Microfilariae, Skin Neoplasms, Wuchereria bancrofti.

\section{Introduction}

Lymphatic filariasis, considered globally as a neglected tropical disease, is a vector borne disease currently endemic in tropical and subtropical Africa, Asia, Western Pacific and part of America affecting over 83 countries. An estimated 600 million people are at risk of lymphatic filariasis infection in 250 endemic districts in 20 states/Union territories in India [1]. In India most widespread lymphatic filariasis infection is due to $W$. bancrofti $(98 \%)$ and the remaining infection by $B$. malayi $(2 \%)$ and bancroftian and brugian filariasis are commonly called bancroftian and brugian filariasis respectively [2]. Culex quinque fasciatus (previously known as Culex fatigans) is the main vector for its spread. Filarial nematodes reside in the sub-cutaneous tissues, deep connective tissues, lymphatic system or body cavities of humans. Lymphatic filariasis is by far the commonest manifestation and is an important public health problem in India. Diagnosis of filarial infection is frequently made on clinical grounds in endemic areas, but demonstration of microfilariae in circulating blood is the only means by which one can make definitive diagnosis $[3,4]$.
We hereby report a case of subcutaneous nodule on the antero-medial aspect of the right arm due to microfilaria.

\section{Case Report}

A 35 years old male presented with a swelling of approximately $3 \times 2.5 \mathrm{~cm}$ on the antero-medial aspect of the right arm since four months [Fig.1]. The swelling was not associated with fever, pain or redness. There was no history of trauma, surgery or travel. A differential diagnosis of lipoma was made. As the patient did not have any signs and symptoms of filarial infection, filariasis was not clinically suspected.

A complete blood count revealed a total count of $6.79 \times 10^{3} / \mu \mathrm{L}$, neutrophils $(58 \%)$, lymphocytes $(30.4 \%)$, monocytes (3.3\%), eosinophils ( $8 \%)$, basophils $(0.3 \%)$, red blood cell count $4.11 \times 10^{6} / \mu \mathrm{L}$ with hemoglobin $14.8 \mathrm{~g} / \mathrm{dL}$. The other investigations such as liver function test, renal function test and blood sugar were normal. HIV test was non-reactive. Ultrasound (USG) of the swelling reported an approximately $5 \mathrm{~cm}$ tubular hypo-echoic lesion in the antero-medial aspect of the right arm communicating with the basilic vein. 
Deep venous system of the arm appeared normal. The lesion was localized only to the subcutaneous plane. Underlying muscle appeared normal. The USG findings were suggestive of the basilic vein thrombophlebitis with a group of lymph nodes compressing the basilic vein.

Fine needle aspiration cytology (FNAC) of the swelling was advised. FNAC of the swelling was done under aseptic conditions, by 23 gauge needle attached to $10 \mathrm{ml}$ disposable syringe. Slides were air dried, fixed, with ether alcohol and stained by haematoxylin and eosin. Fine needle aspiration cytology (FNAC) of the swelling revealed few lymphocytes, occasional eosinophils and cyst macrophages against a background of fluid. Many sheathed microfilariae with central column of nuclei were also noted.

The patient was referred for a DEC (diethylcarbamazine (DEC) provocation test. The patient was advised to take single dose of diethylcarbamazine $100 \mathrm{mg}$ orally to induce appearance of microfilariae (which has nocturnal periodicity), in the peripheral blood in the daytime. Blood sample was collected 30 minutes after diethylcarbamazine (DEC) administration and was examined by three methods. (i) Examination of unstained preparation: Two drops of blood were taken on a clean grease free slide and cover slip was put over it. The slide was examined under low power and then high power objective. Microfilariae were seen as a transparent and sheathed worm with rounded anterior end and pointed tail end [Fig.2]. (ii) Examination of stained preparation: Thin and thick film of blood was prepared. Thick film was dehemoglobinised by immersing the slide in water and intermittent rocking for few seconds. The slide was dried, fixed in methyl alcohol and stained with Leishman stain. Microfilariae were seen in both the thin and thick films. Features like excretory and anal pores and absent body nuclei in tail tip were also observed [Fig.3]. (iii) Knott's concentration method: $1 \mathrm{ml}$ volume of anti-coagulated blood was mixed with $10 \mathrm{ml}$ of $2 \%$ formalin, centrifuging the

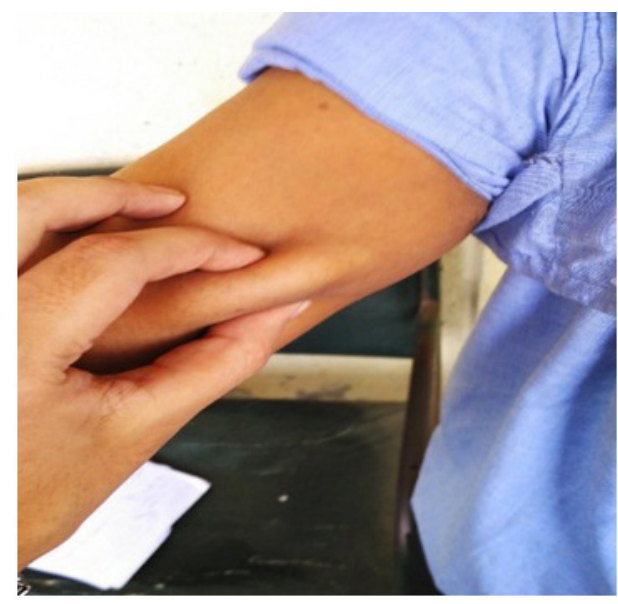

Fig.1: Swelling on the antero-medial aspect of the right arm.

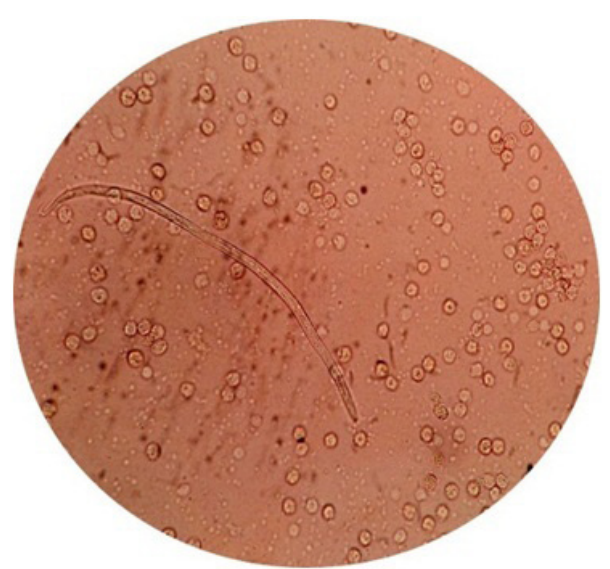

Fig.2: Microfilaria of Wuchereria bancrofti: unstained preparation (wet mount of blood sample low power $10 \times$ ).

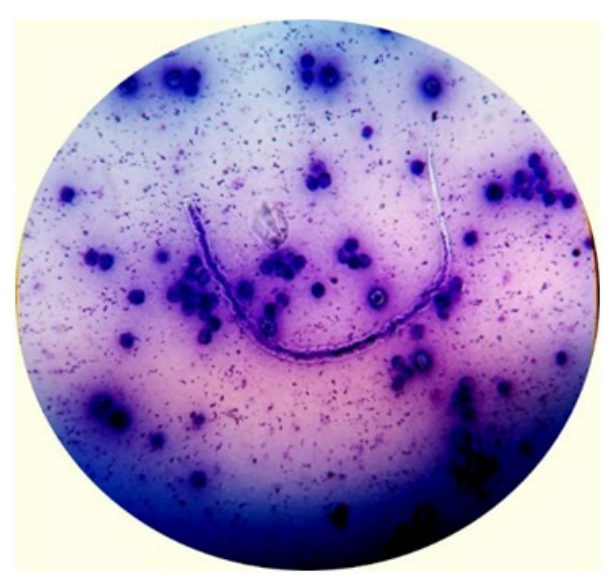

Fig.3: Microfilaria of Wuchereria bancrofti. Stained preparation (Leishman stain, 100×). Note the cephalic and tail tip free from nuclei. 
preparation. The supernatant fluid was decanted and the sediment was drawn into a film which was air dried, fixed and stained with Leishman stain. On examination, microfilaria was detected with features seen as those in the previous method.

$W$. bancrofti was confirmed by absence of nuclei in cephalic and tail end and was thus differentiated from Loa loa. Thus correlating the cytological and microscopic findings it was diagnosed as a case of subcutaneous nodule on the antero-medial aspect of the arm due to filariasis caused by $W$. bancrofti. On follow up, the patient showed improvement with no microfilariae in peripheral blood on repeat DEC provocative test after 2 months.

\section{Discussion}

Filariasis is a major public problem in tropical countries, especially India, China, Indonesia and parts of Africa. Current estimates indicate that there are at least six million attacks of acute filariasis per year and more than 20 million people have one or more chronic filarial lesion. [3]. The international task force for disease eradication has identified lymphatic filariasis as one of the six diseases considered eradicable or potentially eradicable [2]. Clinically, filariasis can be of two major categories, filariasis of skin and subcutaneous tissue and lymphatic filariasis. Onchocerca volvulus and Loa loa are most common organisms reported in former, and Wuchereria bancrofti and Brugia malayi are the two most common species in latter. [5]. In the present case microfilaria of Wuchereria bancrofti was seen in sub-cutaneous swelling in the anteromedial aspect of the right arm.

The life cycle of $W$. bancrofti is found in two hosts. Man is definitive host and mosquito is an intermediate host [6]. Adult worm resides in lymph node where the gravid female releases a large numbers of microfilariae. These larvae pass through the thoracic duct and pulmonary capillaries to the peripheral circulation [7]. The microfilariae of $W$. bancrofti is detected by fine needle aspiration cytology (FNAC) at different sites like breast, thyroid, lymph node, liver, lungs and few cases have been reported in bone marrow and body fluids; but subcutaneous nodule in the arm is a very rare presentation $[6,8,9,10]$ and the appearance of microfilarae in the subcutaneous arm nodule can be considered as a rare site and to best of our knowledge no case has been reported at this site. In our case subcutaneous nodule aspirates showed sheathed microfilariae, $W$. bancrofti was confirmed by absence of nuclei in cephalic and tail end and was thus differentiated from Loa loa. The combined cytological and microbiological findings (DEC provocation test) were useful for the confirmatory diagnosis of microfilarae. DEC is a safe drug, normally used for the treatment of filariasis. For DEC provocation test, $100 \mathrm{mg}$ of DEC is given orally to the patient followed by a blood sample 30-45 minutes later: this procedure can "flush out" microfilariae into the peripheral blood during day time and has a sensitivity that is almost comparable to that of night blood surveys $[11,12]$. Blood collected after $30 \mathrm{~min}$ revealed the microfilariae in unstained as well as stained preparation. Patient was referred to clinician and he responded to diethylcarbamazine $(6 \mathrm{mg} / \mathrm{kg})$ for 21 days.

\section{Conclusion}

In endemic countries like India filariasis should always be considered as a differential diagnosis of swelling at any site. Our presentation revealed that microfilariae may even be present at rare site like subcutaneous nodule on the arm. Cytological as well as DEC provocation test helps to confirm the diagnosis. A prompt and confirmed diagnosis helps in the institution of specific treatment especially in unsuspected cases.

Contributors: SMP: case diagnosis and manuscript writing; AHK: manuscript editing and literature review; PRM: manuscript editing and critical inputs in the manuscript. SMP will act as guarantor of the study. All authors approved the final version of this manuscript.

Funding: None; Competing interests: None stated. 


\section{References}

1. Park E. Epidemiology of communicable diseases. In: Park's textbook of preventive and social medicine. $22^{\text {nd }}$ ed. India: M/s Banarsidas Bhanot: 2013. pp. 245.

2. Sabesan S, Vanamail P, Raju KHK. Lymphatic filariasis in India: Epidemiology and control measures. Journal of Postgraduate Medicine. 2010;56:232-238.

3. Sivakumar S. Role of fine needle aspiration cytology in detection of microfilariae: Report of 2 cases. Acta Cytol. 2007;51:803-805.

4. Rawat V, Rizvi G, Sharma N, Pandey H. An unusual presentation of Wuchereria bancrofti infection. Indian Journal of Medical Microbiology. 2009;27:382-383.

5. Mallick MG, Sengupta S, Bandyopadhyay A, Chakraborty J, Ray S, Guha D. Cytodiagnosis of filarial infections from an endemic area. Acta Cytol. 2007;51:843-849.

6. Arora DR, Arora B, Medical parasitology, $2^{\text {nd }}$ ed. SDR: Delhi; 2005. pp. 184-190.
7. Kishore B, Khare P, Gupta RJ, Bisht SP. Microfilaria of Wuchereria bancrofti in cytologic smears: a report of 5 cases with unusual presentations Acta Cytol. 2008;52:710-712.

8. Valand AG, Pandya BS, Patil YV, Patel LG. Subcutaneous filariasis: An unusual case Report. Indian J Dermatol. 2007;52:48-49.

9. Kumar M, Shukla VK, Gupta S. Incidental detection of microfilariae in cytological smears: Clinical examples. J Trop Med Hyg. 1991;94:110.

10. Dey P, Walker R. Microfilaria in fine needle aspiration from skin nodule. Acta Cyol. 1994;38:114.

11. Manson-Bahr PEC, Wijers DJB. The effect of a small dose of diethylcarbamazine on the circulation of microfilariae of Wuchereria bancrofti. Trans R Soc Trop Med Hyg. 1972;66:18.

12. More SJ, Copeman DB. A highly specific and sensitive monoclonal antibody-based ELISA for the detection of circulating antigen in bancroftian filariasis. Trop Med Parasitol. 1990; 41:403-406. 